\title{
Hacia la estandarización de los perfiles de egreso de los programas de doctorado
}

\author{
Karen Núñez-Valdés* \\ Universidad Autónoma de Chile, Providencia, Chile \\ https://orcid.org/0000-0002-6641-6581 \\ José González Campos \\ Universidad de Playa Ancha, Valparaíso, Chile \\ http://orcid.org/0000-0003-4610-6874
}

\begin{abstract}
Resumen
En este artículo se presenta el análisis de los perfiles de egreso declarados por los programas de Doctorado en Educación dictados en territorio chileno. Este proceso fue realizado en función de un Perfil de Egreso Estándar, donde se explicitan las competencias que ha de adquirir un futuro doctor tras su formación. Este perfil recoge las competencias que se demandan en el contexto globalizado actual, considerando la documentación nacional, internacional y la opinión de los doctorandos. De acuerdo a ello, se propone analizar la coherencia de cada uno de los perfiles de egreso declarados con respecto al perfil de egreso estándar. El método utilizado es de carácter cuantitativo descriptivo, con un diseño de tipo transversal, utilizándose técnicas descriptivas y de asociación. Como resultados se presenta la cuantificación de las competencias disciplinares, metodológicas, instrumentales y genéricas declaradas en los perfiles de egreso de los programas de Doctorado en Educación dictados en Chile, con el fin de contribuir a la evaluación de estos en el contexto nacional. A nivel internacional, se presenta una metodología replicable para el análisis de perfiles de egreso de programas de doctorado.
\end{abstract}

Palabras clave: doctorado; evaluación; metodología; perfiles de egreso

\section{Towards the Standardization of Graduation Profiles in Doctoral Programs}

\begin{abstract}
This article presents the analysis of the graduation profiles declared by the Education Doctoral programs in Chilean territory. This process was carried out in accordance with a Standard Graduation Profile, which spells out the competencies that a future doctor must possess after training. This profile includes the competences required in the current globalized context, taking into consideration national and international documentation, as well as the opinions of the doctoral students. Accordingly, it intends to analyze the consistency of each of the declared graduation profiles with respect to the standard graduation profile. The nature of the method used is descriptive and quantitative, with a cross-sectional design, using descriptive and association techniques. The results include the quantification of the disciplinary, methodological, instrumental, and generic competencies declared in the graduation profiles of the Education Doctoral programs in Chile, with the aim of contributing to their assessment within the national context. At the international level, a replicable methodology is presented for the analysis of the graduation profiles in doctoral programs.
\end{abstract}

Keywords: doctoral; evaluation; methodology; graduation profiles 


\title{
Em busca da padronização dos perfis de egresso de cursos de doutorado
}

\begin{abstract}
Resumo
Este artigo apresenta a análise dos perfis de egresso declarados pelos cursos de Doutorado em Educação ministrados no Chile. Este processo foi realizado em função de um Perfil de Egresso Padrão, onde se explicam as competências que o futuro doutor deve adquirir após a sua formação. Este perfil reúne as competências exigidas no contexto globalizado atual, considerando a documentação nacional e internacional, assim como a opinião dos doutorandos. O objetivo é analisar a coerência de cada um dos perfis de egresso declarados com relação ao perfil de egresso padrão. $O$ método utilizado é o quantitativo descritivo, com um desenho de tipo transversal, que faz uso de técnicas descritivas e de associação. A maneira de resultados, apresenta-se a quantificação das competências disciplinares, metodológicas, instrumentais e genéricas declaradas nos perfis de egresso dos cursos de Doutorado em Educação ministrados no Chile, com o intuito de contribuir para a sua avaliação no âmbito nacional. Igualmente, apresenta-se uma metodologia possível de ser replicada na análise de perfis de egresso de cursos de doutorado, no plano internacional.
\end{abstract}

Palavras-chave: doutorado; avaliação; metodologia; perfis de egresso

Como citar:

Núñez-Valdés, K., \& González, J. (2019). Hacia la estandarización de los perfiles de egreso de los programas de doctorado. Revista Digital de Investigación en Docencia Universitaria, 13(2), 3-18. https://doi.org/10.19083/ridu.2019.1080

L a historia de la educación superior chilena ha sido dividida en cuatro etapas (Bernasconi \& Rojas, 2004), sin embargo, con la aprobación de la Ley de Educación Superior 21.091 se visualiza una quinta etapa, dado los cambios que se introducirán en el financiamiento de la educación superior y los procesos de acreditación institucional y de programas que se proponen. En relación a las etapas, la primera de ellas, se caracterizó por la existencia de un sistema de educación superior pequeño y homogéneo, enfocado especialmente en el pregrado. La segunda, definida a partir de la reforma universitaria y los cambios que trajo consigo esta: el aumento de la matrícula y del financiamiento público, la reorganización de los gobiernos universitarios, la incorporación de académicos con un perfil de investigación y una nueva visión de la vinculación con el medio. Con el Golpe de Estado de 1973 se inició una tercera etapa cuya particularidad está dada por la intervención de las instituciones de educación superior, mediante la designación de rectores militares y la desarticulación de las medidas adoptadas tras la reforma universitaria. La última etapa comenzó con las reformas de 1981 y se caracterizó esencialmente por el drástico descenso de la matrícula en las instituciones de educación superior, en un $30 \%$ aproximadamente, por lo que el gobierno militar autorizó la creación de universidades privadas y de nuevas instituciones no-universitarias de educación superior (institutos profesionales y centros de formación técnica).

Chile cambió de un sistema universitario pequeño, de élite y estatal, hacia un sistema universitario de mercado (Bernasconi \& Rojas, 2004). A la par se rediseñó el sistema de financiamiento de la educación superior, diversificándose el subsidio del Estado. En este contexto, las universidades, a fin de recuperar sus costos de operación iniciaron el cobro de aranceles a sus alumnos, creándose un programa de préstamos estudiantiles para aquellos que no podían financiar sus aranceles. Todos estos cambios se dieron en el marco de los programas de pregrado. En 1982 se creó el Fondo Nacional de Investigación Científica y Tecnológica (FONDECYT) cuyo fin era distribuir fondos de investigación, a través, de la presentación de proyectos los cuales eran arbitrados por pares externos. Recién en la década del 2000, se agregó como tema central a la discusión el postgrado, que había sido escasamente discutido en todo el país (Corvalán, Falabella, \& Rojas, 2011).

La nueva estructura institucional del sistema, forjada a partir de los cambios acaecidos hacia 
1980, permitió que en Chile se incrementara la oferta y por tanto la matrícula universitaria, debiéndose ampliar, además, el sistema hacia la educación de cuarto nivel. En consonancia con las demandas emergentes, se ha reconocido que el conocimiento, en el contexto globalizado, es un componente estratégico en el crecimiento económico de un país (Baeza, 2017; Chiappa, \& Muñoz, 2015). El doctorado representa una instancia de formación de capital humano altamente avanzado, ya que, los programas doctorales permiten avalar que un profesional cuenta con las competencias para el desarrollo de investigaciones originales en un determinado campo de estudio (Comisión Nacional de Acreditación \& Ministerio de Educación, 2007).

Los doctorados constituyen el grado superior que confiere una universidad. El doctorado académico, se caracteriza por el desarrollo de investigaciones rigurosas, las cuales dan cuenta de que una persona domina los conceptos más avanzados en un área del conocimiento y por tanto puede contribuir a un determinado campo del saber (Krauskopf, 2003). A pesar de la importancia que se le ha reconocido a la educación doctoral en Chile, en la década de los noventa se contaba con solo dos programas de Doctorado en Educación, dictados por la Pontificia Universidad Católica de Chile y la Universidad de Humanismo Cristiano, ambos creados el año 1991. El desarrollo tardío del postgrado chileno radicó en que el interés del Estado estuvo centrado en la formación de licenciados y no así en este grado académico (Vera-Villarroel, 2010). Sin embargo, este panorama se ha transformado en función de los cambios de la educación universitaria chilena, lo que se evidencia en el número de programas que actualmente existen. Este fenómeno se explica por el cambio de objetivos que han asumido las universidades, donde el conocimiento es fundamental en el contexto globalizado (Weijden, Teelken, de Boer, \& Drost, 2016). De este modo, las universidades han debido hacerse cargo de las demandas de la sociedad de la información, donde el conocimiento es considerado un bien económico (Fernández, Fastuca, \& Wainerman, 2015). En el año 2018, se dictaron en Chile un total de 20 programas de Doctorado en Educación, definidos por área de estudios (Tabla 1), según los datos entregados por el Ministerio de Educación (2018b).

Tabla 1

Doctorados en Educación dictados en Chile al año 2018.

\begin{tabular}{|l|l|}
\hline Universidad & Nombre del Programa \\
\hline 1. $\quad$ Pontificia Universidad Católica de Chile & Doctorado en Ciencias de la Educación \\
\hline 2. $\quad$ Pontificia Universidad Católica de Valparaíso & Doctorado en Didáctica de la Matemática \\
\hline 3. $\quad$ Universidad Academia Humanismo Cristiano & Doctorado en Educación \\
\hline 4. $\quad$ Universidad Alberto Hurtado & Doctorado en Educación \\
\hline 5. $\quad$ Universidad Andrés Bello & Doctorado en Educación y Sociedad \\
\hline 6. $\quad$ Universidad Bernardo O'Higgins & Doctorado en Educación \\
\hline 7. $\quad$ Universidad Católica de la Santísima Concepción & Doctorado en Educación \\
\hline 8. $\quad$ Universidad Católica de Temuco & Doctorado en Educación \\
\hline 9. $\quad$ Universidad Católica del Maule & Doctorado en Educación \\
\hline 10. $\quad$ Universidad Católica del Maule & Doctorado en Ciencias de la Act. física \\
\hline 11. $\quad$ Universidad Católica del Maule & Doctorado en Didáctica de la Matemática \\
\hline 12. $\quad$ Universidad de Concepción & Doctorado en Educación \\
\hline 13. $\quad$ Universidad de la Frontera & Doctorado en Ciencias de la Educación \\
\hline 14. $\quad$ Universidad de los lagos & Doctorado en Ed. Matemática \\
\hline 15. $\quad$ Universidad de Santiago de Chile & Doctorado en Ciencias de la Educación \\
\hline 16. $\quad$ Universidad del Biobío & Doctorado en Educación \\
\hline 17. $\quad$ Universidad Diego Portales & Doctorado en Educación \\
\hline 18. $\quad$ Universidad Diego Portales & Doctorado en Educación Superior \\
\hline 19. $\quad$ Universidad Metropolitana de Ciencias de la Educación & Doctorado en Educación \\
\hline 20. $\quad$ Universidad SEK & Doctorado en Educación \\
\hline
\end{tabular}

Nota. Elaborado a partir del Sistema Nacional de Información para la Educación Superior (Ministerio de Educación, 2018b) y páginas web de las universidades. 
De los programas catastrados, el doctorado de la Universidad Academia Humanismo Cristiano señala en su página web que está sin ingreso para el presente año, en tanto el programa de la Universidad de Santiago de Chile se encuentra cerrado al año 2019. Cabe señalar, que los programas de las universidades Diego Portales y Alberto Hurtado se dictan en conjunto, por lo que comparten el perfil de egreso y las actividades formativas. Lo mismo acontece con el Programa de Doctorado dictado en Consorcio por la Universidad Católica de la Santísima Concepción, la Universidad Católica de Temuco, la Universidad Católica del Maule y la Universidad del Biobío, las que constituyen un solo programa doctoral. En síntesis, para el año 2019 la oferta real de programas de Doctorado en Educación es de 14. Es relevante señalar que, de los programas existentes, el Doctorado en Educación Superior de la Universi- dad Diego Portales posee una doble titulación, ya que es impartido conjuntamente con la Facultad de Humanidades de la Universidad de Leiden, Holanda. El Doctorado en Educación de la Universidad Bernardo O'Higgins, en tanto, mantiene un Convenio Marco de colaboración académica con la Universidad de Valencia, España.

Considerando la oferta de programas de Doctorado en Educación para el año 2018, la matrícula a nivel nacional fue de 321 estudiantes, ingresando al primer año 76 aspirantes, lo que corresponde al $24 \%$ del total de los matriculados (Tabla 2). Esta situación se contrasta con años precedentes, por ejemplo, la matrícula nacional para el año 2009 fue de 524 estudiantes (Corvalán et al., 2011). La cifra actual representa un $38 \%$ menos de estudiantes matriculados en programas de Doctorado en Educación en Chile, en un período menor a una década.

Tabla 2

Matrícula total y del primer año en programas de Doctorado en Educación en Chile presentados por universidad al año 2018.

\begin{tabular}{|c|c|c|}
\hline Universidad & Matriculados & $\begin{array}{c}\text { 1er año } \\
2018\end{array}$ \\
\hline 1. Pontificia Universidad Católica de Chile & 49 & 12 \\
\hline 2. Pontificia Universidad Católica de Valparaíso & 28 & 6 \\
\hline 3. Universidad Academia Humanismo Cristiano & 19 & 7 \\
\hline 4. Universidad Alberto Hurtado & 8 & 2 \\
\hline 5. Universidad Andrés Bello & 6 & 6 \\
\hline 6. Universidad Bernardo O’Higgins & 7 & 0 \\
\hline 7. Universidad Católica de la Santísima Concepción & 13 & 4 \\
\hline 8. Universidad Católica de Temuco & 12 & 3 \\
\hline 9. Universidad Católica del Maule & 20 & 3 \\
\hline 10. Universidad Católica del Maule & 6 & 0 \\
\hline 11. Universidad Católica del Maule & 3 & 3 \\
\hline 12. Universidad de Concepción & 7 & 0 \\
\hline 13. Universidad de la Frontera & 6 & 0 \\
\hline 14. Universidad de los lagos & 11 & 5 \\
\hline 15. Universidad de Santiago de Chile & 14 & 0 \\
\hline 16. Universidad del Biobío & 12 & 4 \\
\hline 17. Universidad Diego Portales & 8 & 3 \\
\hline 18. Universidad Diego Portales & 19 & 3 \\
\hline 19. Universidad Metropolitana de Ciencias de la Educación & 12 & 8 \\
\hline \multirow[t]{2}{*}{ 20. Universidad SEK } & 61 & 7 \\
\hline & 321 & 76 \\
\hline
\end{tabular}

Nota. Elaborado a partir del Sistema Nacional de Información para la Educación Superior (Ministerio de Educación, 2018b) y páginas web de las universidades. 


\section{Acreditación de los programas de doctorado en Chile.}

Los procesos de acreditación en Chile tienen sus antecedentes en la década de los ochenta, donde el nuevo contexto, a saber, la creación de universidades, institutos profesionales y centros de formación técnica estaban bajo un sistema de supervisión que tenía por objetivo revisar los planes de estudio de las nuevas carreras (Bernasconi \& Rojas, 2004). Este sistema de supervisión colapsó a fines de la década de los ochenta, debido a la excesiva carga de examinaciones y al relajo de los estándares aplicados, lo que supuso la pérdida de credibilidad de este. En el año 1990 se dictó la ley N¹8.962 - Ley Orgánica Constitucional de Enseñanza (LOCE)- la que estableció un nuevo mecanismo de supervisión a las instituciones de carácter privadas, basado en la acreditación por parte de un Consejo Superior de Educación (CSE), autónomo y de carácter predominantemente técnico. Si bien el nuevo sistema recibió el nombre de acreditación este difiere de lo que usualmente se entiende por ella, por lo que más tarde el Ministerio de Educación comenzaría a llamarlo licenciamiento (Bernasconi \& Rojas, 2004).

Es de relevar, que los procesos de acreditación en los años noventa estaban focalizados solo en las instituciones privadas, siendo esta experiencia el inicio de la cultura evaluativa en Chile. A fines de esa década, en el año 1999, se creó la Comisión Nacional de Acreditación de Pregrado (CNAP). En lo referido a la acreditación de programas de postgrados, la Comisión de Evaluación de la Calidad de Programas de Postgrado (CONAP) fue la encargada de liderar los procesos de acreditación, ya que contaba con una década de experiencia bajo el alero de la Comisión Nacional de Investigación Científica y Tecnológica (CONICYT) realizando evaluaciones a los programas para efectos de otorgar becas a sus estudiantes (Bernasconi \& Rojas, 2004). Esta comisión determinó que a aquellos programas que no cumplieran con los parámetros mínimos de aseguramiento de la calidad, se les podía negar la acreditación (Comisión Nacional de Acreditación \& Ministerio de Educación, 2007).

El año 2006, se promulgó la Ley de Aseguramiento de la Calidad de la Educación N²0.129, entrando al año siguiente en funcionamiento un sis- tema integrado de aseguramiento de la calidad de la educación. De este modo, se fusionan la CONAP y la CNAP en la Comisión Nacional de Acreditación (CNA), quien institucionaliza la acreditación en la educación superior chilena (Comisión Nacional de Acreditación \& Ministerio de Educación, 2007).

De este modo, la CNA asumió las funciones de acreditación institucional y de los programas de pregrado y de postgrado, estableciéndose, a través de la ley, las bases institucionales para la creación del sistema de evaluación actualmente vigente en Chile (Espinoza \& González, 2016).

La acreditación de programas doctorales fue establecida por ley, a través de la autoevaluación y la heteroevaluación de pares externos, aplicándose dimensiones y criterios de evaluación para esto (González, Sarzoza \& López, 2018).

El año 2010 con la dictación de la Ley General de Educación (LGE) se reemplazó la Ley Orgánica Constitucional de Enseñanza (LOCE), pasando la regulación de la educación superior a manos del Consejo Nacional de Educación (CNED), quien se convirtió en la entidad responsable del Sistema de Aseguramiento de la Calidad de la Educación, reemplazándose con ello al Consejo Superior de Educación (CSE). Con este cambio se estableció que los procesos de acreditación serían voluntarios, salvo excepciones como las carreras de educación y medicina, y que las etapas de acreditación serían evaluación interna, evaluación externa y juicio de acreditación (Cancino \& Schmal, 2014).

Tras la puesta en marcha de la CNA, en el año 2014 se aprobó el Reglamento que fijó el procedimiento para el desarrollo de los procesos de acreditación de postgrado y especialidades del área de salud. Este reglamento contempló a los programas de magíster, doctorado y especialidades en el área de la salud y de otros niveles equivalentes. Tanto el año 2016 como en el año 2018 se realizaron modificaciones a este reglamento, quedando establecidos los elementos constitutivos de los procesos de acreditación de este nivel (Comisión Nacional de Acreditación, 2016a, 2018).

En la actualidad, el aseguramiento de la calidad, a través de los procesos de acreditación se constituye como una herramienta de regulación de la educación superior, cuyo objetivo es la certificación de la calidad de los programas ofrecidos 
en Chile. En términos de postgrado es importante señalar que, a pesar de la institucionalidad existente, aún hay una porción relevante de programas que no se encuentran acreditados, lo que evidencia el carácter laxo del marco regulatorio del nivel doctoral (Baeza, 2017), situación que se pretende subsanar con la aprobación de la Ley 21.091 en la que se establece que las universidades deberán someter sus programas de doctorado a los procesos de acreditación y su acreditación será requisito para acceder a financiamiento público o para contar con la garantía del Estado (Ministerio de Educación, 2018a). Lo que implica que si un programa de doctorado no se encuentra acreditado no será reconocido por el Estado y en consecuen- cia sus estudiantes no podrán acceder a financiamiento público de ningún tipo. El financiamiento estatal para este nivel se gestiona por medio del Programa Becas Chile, creado el año 2008, y que surge por la necesidad de contar con profesionales de alto nivel que adquieran las competencias necesarias para el desarrollo del país (González \& Jiménez, 2014). Esta situación se presenta para los programas de Doctorado en Educación como extremadamente crítica, considerando el número de programas actualmente acreditados (véase Tabla 3) lo que representa todo un reto para las universidades, considerando que el financiamiento es fundamental para la formación de un doctorando de cara a la investigación (Horgué, 2012):

Tabla 3

Doctorados en Educación chilenos acreditados al año 2019.

\begin{tabular}{|c|c|c|c|}
\hline Universidad & $\begin{array}{c}\text { Estado de } \\
\text { Acreditación }\end{array}$ & $\begin{array}{c}\mathbf{N}^{\circ} \text { años } \\
\text { Acreditación }\end{array}$ & Expiración \\
\hline 1. Pontificia Universidad Católica de Chile & Acreditado & 4 años & 2021 \\
\hline 2. Pontificia Universidad Católica de Valparaíso & Acreditado & 3 años & 2019 \\
\hline 3. Universidad Academia Humanismo Cristiano & No & & \\
\hline 4. Universidad Alberto Hurtado & Acreditado & 4 años & 2021 \\
\hline 5. Universidad Andrés Bello & No & & \\
\hline 6. Universidad Bernardo O'Higgins & No & & \\
\hline 7. Universidad Católica de la Santísima Concepción & Acreditado & 3 años & 2019 \\
\hline 8. Universidad Católica de Temuco & Acreditado & 3 años & 2019 \\
\hline 9. Universidad Católica del Maule & Acreditado & 3 años & 2019 \\
\hline 10. Universidad Católica del Maule & No & & \\
\hline 11. Universidad Católica del Maule & No & & \\
\hline 12. Universidad de Concepción & No & & \\
\hline 13. Universidad de la Frontera & No & & \\
\hline 14. Universidad de los lagos & No & & \\
\hline 15. Universidad de Santiago de Chile & No & & \\
\hline 16. Universidad del Biobío & Acreditado & 3 años & 2019 \\
\hline 17. Universidad Diego Portales & Acreditado & 4 años & 2021 \\
\hline 18. Universidad Diego Portales & No & & \\
\hline 19. Universidad Metropolitana de Ciencias de la Educación & No & & \\
\hline 20. Universidad SEK & No & & \\
\hline
\end{tabular}

Nota. Elaborado a partir del Sistema Nacional de Información para la Educación Superior (Ministerio de Educación, 2018b) y páginas web de las universidades. 
De la Tabla 3, se desprende que, 8 doctorados en educación se encuentran acreditados, lo que equivale al $40 \%$ del total de la oferta chilena. Es importante indicar que, si consideramos que existen programas dictados en consorcio y en conjunto, la cifra decae a 4 programas, siendo estos los dictados por la Pontificia Universidad Católica de Chile, la Pontificia Universidad Católica de Valparaíso, el Consorcio de Universidades y la Universidad Alberto Hurtado/Universidad Diego Portales. Otro elemento no menor, es que la acreditación de estos programas está entre 3 y 4 años, siendo el máximo 10 años de acreditación. Los programas que obtuvieron 3 años se encuentran en el primer tramo determinado por la Comisión Nacional de Acreditación, en el que se señala que el programa presenta deficiencias que se deben mejorar (Comisión Nacional de Acreditación, 2016b).

Por su parte, los programas que obtuvieron 4 años se encuentran en el tercer tramo, definido como aquel programa que cumple con los aspectos del criterio de buena forma, aunque se requieren algunas mejoras (Comisión Nacional de Acreditación, 2016b). Ambos tramos poseen una distancia notoria con el cuarto y máximo período de acreditación, que se ubica entre 8 y 10 años, el cual señala que el programa cumple de forma sobresaliente con todos los aspectos que son evaluados, siendo mínimas sus debilidades (Comisión Nacional de Acreditación, 2016b).

En tanto, si consideramos la importancia de la acreditación como una forma de regulación de los programas doctorales, queda en evidencia que el $60 \%$ de estos funcionan sin una certificación de calidad, por lo que el $49 \%$ de los estudiantes matriculados estudia en programas no acreditados, específicamente 160 doctorandos para el año 2018 lo que, además, significa que estos no tienen la posibilidad de acceder a financiamiento por parte del Estado para cursar estudios doctorales, ya que es requisito de postulación estar matriculado en un programa acreditado.

\section{Del perfil egreso como evaluación de los programas doctorales.}

Bien es sabido, que la globalización ha tenido un gran impacto a todo nivel, siendo uno de ellos la educación superior, la que ha debido adaptarse a los nuevos desafíos que surgen con motivo de la inmediatez y el intercambio de productos, de capital humano y de recursos. Esto ha ocurrido en función de la rapidez con que se realizan los intercambios comerciales y las comunicaciones (Núñez Valdés, 2017), traduciéndose en un cambio de paradigma que incorpora el concepto de competencias, el cual se basa en una concepción educativa donde la relación entre saberes complejos y su profundización es esencial para que los sujetos logren integrarlos en el mundo laboral (Muñoz \& Araya, 2017).

Las competencias son definidas como un saber complejo que permite la movilización de diferentes habilidades, conocimientos y actitudes (Villarroel \& Bruna, 2014). Dada la significatividad de estas, las universidades han debido incorporar en sus programas formativos y en sus perfiles de egreso las competencias que han de adquirir los aspirantes a un determinado grado académico.

El perfil de egreso se constituye así, en una declaración formal de una institución de educación superior sobre las competencias que ha de adquirir un estudiante tras su formación. Es el compromiso que se adquiere para con la sociedad, ya que, es en él donde se dan a conocer los procesos formativos y las competencias claves para una determinada profesión (Hawes, 2010). Möller y Gómez (2014, p.25) definen que el "perfil de egreso (como definición identitaria y como compromiso formativo), se concibe como un instrumento que dota de sentido a los programas de formación, por lo que resulta fundamental que estos incluyan mecanismos para evaluar su cumplimiento".

Las competencias que se han identificado en el perfil de egreso se constituyen como un insumo de evaluación tanto de los procesos formativos como del desempeño de un determinado estudiante (Knust \& Gómez, 2009). De este modo, el perfil de egreso da cuenta del tipo de graduado que formará una institución de educación superior y que se insertará posteriormente en el mundo laboral.

A nivel internacional, y más específicamente en Europa, se entiende que un programa doctoral se constituye como el tercer ciclo de estudios universitarios y conduce a la adquisición de com- 
petencias y habilidades relacionadas con la investigación científica de calidad (Horgué, 2012). Tras este reconocimiento y el desarrollo del proceso de Bolonia- iniciado hace veinte años- se comienza a entender que la universidad del siglo XXI deberá centrarse en la formación doctoral, lo que supone un trabajo interdisciplinario, cooperativo e internacional. De este modo el doctorado es la marca de la Universidad investigando (Nebot, 2009).

Esta nueva visión de universidad, derivó en múltiples discusiones en torno a la formación doctoral, estableciéndose entre otras cosas, las competencias que debía adquirir un doctor y que serían consagradas en los perfiles de egreso de cada uno de los programas dictados en el espacio europeo. En consecuencia, se releva la importancia de la formación doctoral, ya que se reconoce como fundamental para el desarrollo de la investigación (Jiménez-Ramírez, 2017). Un perfil de egreso debe contener no sólo una definición del tipo de programa que se impartirá, sino que además los atributos que desarrollarán los doctorandos en el proceso formativo (Spronken-Smith, Brown \& Mirosa, 2018)

En Chile, en tanto, no se han determinado el grupo de competencias que debe adquirir un doctor con su formación. Sin embargo, se entiende que un programa doctoral está vinculado a la formación de investigadores (Corvalán et al., 2011), no existiendo una definición del tipo de investigador que se formará. En este contexto Núñez-Valdés y González (2019) elaboraron un perfil de egreso denominado Perfil de Egreso Estándar que reúne las competencias que debe adquirir un futuro doctor en su proceso de formación. Los autores utilizaron como insumos para su propuesta los documentos elaborados desde el Proceso de Bolonia hasta la actualidad (nivel internacional), los documentos emanados desde la Comisión Nacional de Acreditación (Chile) y la opinión de los doctorandos sobre las competencias que deberían adquirir al finalizar su formación doctoral. Del análisis de esta tríada se desprende el Perfil de Egreso Estándar para los programas de doctorado chilenos:

\section{Perfil de Egreso Estándar para el grado académico de Doctor en Educación.}

Un graduado con el grado de Doctor en Educación, demostrará habilidades altamente desa- rrolladas en la definición, gestión y comunicación de investigaciones originales en un área especializada.

\section{Definición del campo de estudio}

Un doctor en educación realizará una contribución significativa al conocimiento en su campo de especialización, por lo que, al completar sus estudios, será capaz de:

- Realizar investigación de la más alta calidad que aporte al conocimiento disciplinar tanto a nivel nacional como internacional.

- Desarrollar su línea de investigación con el fin de contribuir a la comunidad científica a la cual pertenece

- Dominar tanto metodologías cuantitativas como cualitativas, de tal forma de realizar la elección de esta en función de los objetivos propuestos para una determinada investigación.

Evaluar y aplicar las técnicas de investigación cuantitativas y cualitativas con el fin seleccionar aquella que aborde con mayor comprensión los fenómenos a investigar.

Los doctores en Educación poseerán las siguientes competencias transferibles:

- Pensamiento crítico.

- Ética.

- Trabajo en equipo.

- Liderazgo.

Fuente: Núñez-Valdés y González (2019, p. 173).

En virtud de la necesidad de conocer el tipo de investigador que se está formando en Chile y de las competencias que ha de adquirido tras su formación doctoral, se propone analizar los perfiles de egreso declarados por los programas de doctorado en educación chilenos -dictados hasta el año 2018- en función del Perfil de Egreso Estándar (Núñez-Valdés \& González, 2019) para así establecer si existe coherencia entre estos. Para el desarrollo de esta tarea se han planteado los siguientes objetivos de investigación:

Objetivo 1: Determinar las similitudes entre el perfil de egreso estándar y los perfiles de doctorado en educación chilenos. 
Objetivo 2: Evidenciar las áreas de estudio predominantes en los programas de doctorado en educación en Chile.

Objetivo 3: Cuantificar porcentualmente las frecuencias de aparición de las competencias en los perfiles de egreso de los programas de doctorado, a saber, competencias disciplinares, metodológicas, instrumentales y genéricas.

Objetivo 4: Jerarquizar las competencias disciplinares predominantes en los perfiles de egreso de los programas de doctorado en educación chilenos.

\section{Método}

Este estudio es de carácter cuantitativo descriptivo. El diseño de investigación es de tipo transversal. Se analizaron diferentes documentos (perfiles de egreso) sobre un mismo contenido (competencias), utilizándose técnicas descriptivas y de asociación (Silverman et al., 2018).

La muestra, serán todos los perfiles de egreso declarados por los programas de Doctorado en Educación de las universidades chilenas vigentes al año 2018.

Cada unidad muestral será recolectada desde las distintas páginas web de las universidades que imparten estos programas (17 universidades). Los perfiles de egreso de los doctorados, se encuentran disponibles para el público general, por lo que no existen restricciones éticas para su recolección.

El software a utilizar para el análisis de los datos será el R-project, en su versión 3.5.1, y el programa Excel 2010.

\section{Resultados}

El perfil de egreso estándar se ha organizado en función del área de estudio y de las competencias presentes en él, por lo que los análisis se basan en: 1) definición del campo de estudio del programa, 2) declaración de competencias disciplinares, 3) competencias metodológicas, 4) competencias instrumentales y 5) competencias genéricas.

Uno de los primeros hallazgos de este estudio, es la existencia de programas doctorales que no utilizan la denominación de perfil de egreso para la explicitación del compromiso formativo que han adquirido para con los aspirantes al grado de Doctor, sino que se refieren a esta declaración como Objetivos del Programa. Del total de 20 programas, 3 de estos hacen alusión a Objetivos y no a Perfil de Egreso. De igual modo, al revisar cada uno de los perfiles presentados por los distintos programas e instituciones, resalta la heterogeneidad entre ellos, tanto en su redacción como en su contenido.

En términos de la "Definición del Campos de estudio", 19 de los 20 programas realizan una declaración explicita de este, lo que equivale a un 95\% del total de la oferta actual en el área. Cabe señalar que el programa que no hace alusión al campo de estudio, solo señala que se trabajará en función de las líneas de investigación del programa, no obstante, no hay información clara con respecto a ellas en la página web de la institución.

En relación a la declaración de las "Competencias Disciplinares" el 100\% de los programas dan cuenta de ellas, oscilando su explicitación entre dos y siete competencias, según cada institución. Por su parte las "Competencias Metodológicas" solo son explicitadas por 3 programas lo que equivale al 15\% del total de la oferta doctoral, dejando en evidencia que no son un elemento constitutivo de los perfiles de egreso de los programas de doctorado en educación chilenos, en consecuencia, distanciándose del perfil de egreso estándar. Lo mismo ocurre en términos de las "Competencias Instrumentales" las cuales son declaradas solo en un programa de doctorado, por lo que estas no son consideradas en la elaboración de los perfiles de egreso, lo cual refleja las eventuales ausencias de los procesos de formación doctoral en Chile.

Las "Competencias genéricas" por su parte, se presentan en 9 de los 20 programas ofertados, lo que equivale a un $45 \%$ del total de estos, en un grupo estas se presentan en función de los objetivos mismos del programa y en otros en relación a las competencias transferibles que declara la institución a nivel general.

Un elemento que no se consideró en el perfil de egreso estándar y que emergió de los análisis, es la variable "Campo laboral en el que se pueden 
desempeñar los futuros doctores", la cual aparece en 9 de los 20 programas examinados, lo que equivale al $45 \%$ del total de la oferta doctoral actual, lo que demuestra la importancia que le otorgan las instituciones al conocimiento por parte de los aspirantes al grado de Doctor de los posibles campos labores a integrarse al culminar su formación doctoral.

En la Tabla 4 se presentan las áreas de estudio declaradas por los programas de Doctorado en Educación ofrecidos en Chile.

Según lo expuesto en la Tabla 4, existe diversidad en términos de las áreas de estudios en la oferta doctoral chilena. El área Investigación educativa interdisciplinaria y pluridisciplinaria es la que concentra el mayor número de programas, lo cual acontece porque esta área ha sido declarada por las cuatro universidades que conforman el Consorcio de universidades y dictan el programa doctoral en conjunto. Llama la atención que no exista especificidad al declarar las áreas de estudio, dejando un amplio espectro para los trabajos de investigación. Los programas dedicados a la didáctica de la matemática son los más específicos en relación a su línea de investigación, así también, los programas que han declarado el curriculum y la educación intercultural como su área específica de investigación. Con respecto a los otros programas, y para conocer con mayor certeza su área, se debería indagar en las líneas de investigación que poseen para establecer claramente cuál es el área de estudio que ofrecen.

En cada perfil de egreso se analizaron las competencias disciplinarias declaradas por los programas de doctorado. Estas se agruparon según sus coincidencias y se establecieron las frecuencias de ellas. En la Tabla 5 se presentan las 26 competencias disciplinarias cuantificadas en los perfiles de egreso.

\section{Tabla 4}

Áreas de estudios de los programas doctorales del área de educación.

\begin{tabular}{|l|c|}
\hline Área de estudio & $\begin{array}{c}\text { No programas } \\
\text { que se identifican } \\
\text { con el área }\end{array}$ \\
\hline Comprensión de la Educación & 1 \\
\hline Didáctica de la Matemática & 2 \\
\hline Práctica educativa & 1 \\
\hline Mejoramiento de políticas públicas y del sistema escolar & 2 \\
\hline Educación y Sociedad & 1 \\
\hline Líneas de investigación del programa (no presente en la página web de la institución) & 1 \\
\hline Investigación educativa interdisciplinaria y pluridisciplinaria & 4 \\
\hline Educación Superior & 1 \\
\hline Fenómeno educativo & 2 \\
\hline Ciencias de la Actividad física & 1 \\
\hline Educación Intercultural & 1 \\
\hline Ciencias de la Educación & 1 \\
\hline Curriculum & 1 \\
\hline Educación Matemática & 1 \\
\hline
\end{tabular}


Tabla 5

Competencias disciplinarias de los Perfiles de Egreso de los Programas de Doctorado en Educación chilenos.

\begin{tabular}{|c|c|c|}
\hline Competencias Disciplinares & $f$ & $\%$ \\
\hline Realizar investigaciones autónomas & 4 & 15 \\
\hline Concebir investigación de alto nivel & 7 & 27 \\
\hline Perspectiva crítico-transformadora & 1 & 4 \\
\hline Desarrollo de investigación avanzada & 6 & 23 \\
\hline Sólidos conocimientos teóricos y prácticos sobre educación & 14 & 54 \\
\hline Liderar equipos de investigación & 5 & 19 \\
\hline Comprender líneas de pensamiento y acción pedagógica & 1 & 4 \\
\hline Interpretar teorías y paradigmas de otras disciplinas & 1 & 4 \\
\hline Poseer rigurosidad epistemológica y metodológica & 1 & 4 \\
\hline Reflexión sistemática & 2 & 8 \\
\hline Capacidad de analizar un problema educativo en profundidad & 2 & 8 \\
\hline Enfoque interdisciplinario & 6 & 23 \\
\hline Generar publicaciones en revistas especializadas & 10 & 38 \\
\hline Analizar desde una crítica epistemológica las nuevas ideas & 4 & 15 \\
\hline Creatividad en los procesos Metodológicos & 1 & 4 \\
\hline Generación de políticas publicas & 1 & 4 \\
\hline Proponer y llevar a cabo proyectos de investigación & 10 & 38 \\
\hline Aplicar el conocimiento propio del área de estudio & 1 & 4 \\
\hline Capacidad de analizar un problema educativo en profundidad & 3 & 12 \\
\hline Vigilancia epistemológica & 1 & 4 \\
\hline Resolver problemas educacionales & 1 & 4 \\
\hline Proponer alternativas científicas & 1 & 4 \\
\hline Colaborar con distintos actores del área & 1 & 4 \\
\hline Promover el desarrollo de espacios y núcleos de debates & 1 & 4 \\
\hline Realizar docencia en diversas instancias & 1 & 4 \\
\hline Aportar a la innovación & 2 & 8 \\
\hline
\end{tabular}

De las competencias declaradas, la más importante, es la referida a los sólidos conocimientos teóricos y prácticos sobre educación, ya que ha sido parte de 14 perfiles de egreso, lo que constituye un 54\%, denotando la relevancia que se le otorga al conocimiento del área de estudio, que en este caso es la educación. En consonancia con las demandas actuales para la investigación, la competencia referida a generar publicaciones en revistas especializadas se posiciona en segundo lugar, lo que implícitamente refiere a la competencia de escritura académica señalada por los doctorandos, la cual es considerada como fundamental por ellos, dado su futuro como investigadores. 
La misma posición ocupa la competencia referida a proponer y llevar a cabo proyectos de investigación, lo que resulta indispensable para el desarrollo de investigación de alto impacto y además para acceder a financiamiento público (por ejemplo, proyectos financiados, a través, FONDECYT). Cabe destacar que existe hetero- geneidad en las competencias declaradas en los perfiles de egreso, pues muchas de ellas se enfocan más en actividades que en competencias. A continuación, se presenta la figura número uno donde se jerarquizan las competencias disciplinares según su frecuencia en los perfiles de egreso:
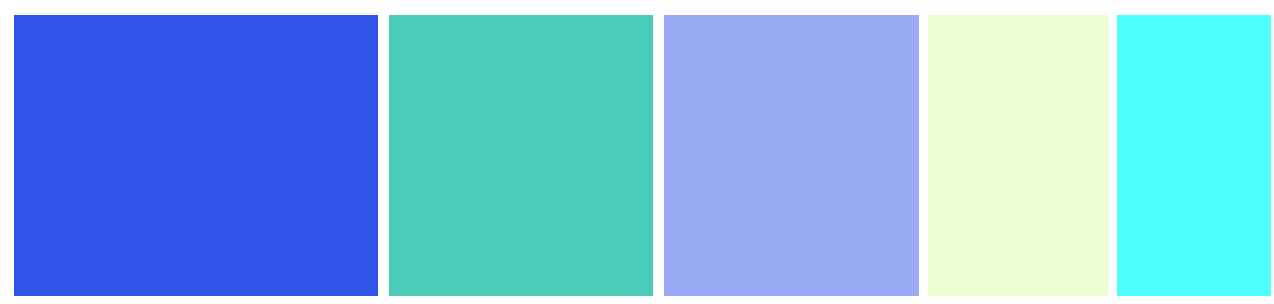

Sólidos conocimientos teóricos y prácticos sobre educación

Generar publicaciones en revistas especializadas

Proponer y llevar a cabo proyectos de investigación

Concebir investigación de alto nivel

Desarrollo de investigacieon avanzada

Enfoque interdisciplinario

Figura 1: Jerarquización de las competencias disciplinares según su frecuencia en los perfiles de egreso.

En términos, de competencias metodológicas solo 3 programas hacen referencia a estas, en consecuencia, no se evidencia un compromiso formativo por parte de las instituciones con respecto a ellas, ya que no son declaradas en sus perfiles de egreso. Esta situación resulta llamativa, dado que, a nivel internacional y nacional, específicamente en la opinión de los doctorandos, se le entrega gran importancia a la formación metodológica en un programa doctoral.

En lo referido a las competencias instrumentales, solo un programa chileno declara estas en sus perfiles de egreso, representando una debilidad al comparar estos con los requerimientos actuales de la investigación a nivel mundial.

Las competencias genéricas o también llamadas transferibles son declaradas por 9 programas, lo que equivale al $45 \%$ del total de la oferta nacional. En algunos perfiles de egreso se menciona más de una de estas competencias. La ética es la competencia que más se repite en los perfiles analizados, pues es tratada como fundamental en la labor investigativa. Otras instituciones otorgan relevancia a aquellas competencias que se alienan con las competencias declaradas a nivel institucional.

Un elemento que no fue considerado, ya que no es parte del perfil de egreso estándar con el cual se analizaron los perfiles doctorales es el campo laboral. Este fue señalado por 9 programas doctorales, dándole gran énfasis en el perfil de egreso al futuro laboral del aspirante a doctor. La línea de trabajo, considerada como la más importante para el graduado de un Doctorado en Educación, es la docencia universitaria y no así la investigación, la cual es el objetivo de un programa doctoral, considerando la definición de este grado académico. 
Los programas de doctorado acreditados en Chile se encuentran en los dos primeros tramos establecidos por la Comisión Nacional de Acreditación, por lo que presentan falencias y/o elementos a mejorar, en algunos de los criterios que son evaluados por esta entidad. En este sentido, al analizar los perfiles de egreso de estas instituciones se evidencian coincidencias con el perfil de egreso estándar presentado, no obstante, estos no cumplen con la totalidad de los elementos constitutivos de este. Con respecto al área de estudio, todos estos perfiles dan cuenta de él, lo mismo ocurre con las competencias disciplinares, dado que cada programa detalla estas, variando entre 4 y 7 competencias dependiendo de la institución. En lo referido a las competencias metodológicas ninguno de los programas acreditados hace alusión a estas en sus perfiles de egreso, siendo por tanto una competencia ignorada en la declaración formativa del programa doctoral. Lo mismo ocurre con las competencias instrumentales, las cuales no son parte de los perfiles analizados. En tanto, las competencias genéricas son declaradas por 6 de los 8 programas doctorales acreditados.

\section{Discusión}

Los cambios acaecidos en la educación superior chilena en los últimos años dejan en evidencia que se inicia un nuevo periodo en la historia de esta, pues con la aprobación y pronta implementación de la Ley 21.091 no solo se establecen las bases para el acceso a fondos del Estado para las instituciones dedicadas a este nivel educativo, sino también un marco regulatorio más exigente para los programas de doctorado dictados en el territorio nacional (Ministerio de Educación, 2018a). El gran cambio presentado en esta ley es la obligatoriedad de acreditación de los programas de doctorado chilenos para contar con la garantía del Estado, por tanto, será a través de los procesos de acreditación que se espera regular el carácter laxo que hoy estos evidencian, especialmente en el área de educación (Baeza, 2017; Corvalán, et. al, 2011)

La necesidad de contar con perfiles de egreso capaces de dar cuenta de una formación sólida a nivel doctoral, en torno a las competencias disci- plinares, metodológicas, instrumentales y genéricas, se hace cada vez más importante, pues un doctor es capital humano avanzado vital para el crecimiento y desarrollo económico de un país, por lo que hacerse cargo de ese nivel es fundamental de cara a las demandas de la sociedad globalizada (Baeza, 2017; Jiménez-Ramírez, 2017).

La propuesta de un perfil de egreso estándar, nace de una reflexión, en torno a tres elementos, el primero de ellos fueron los cambios vividos en Europa desde el Proceso de Bolonia, iniciado hace dos décadas, cuando se toma conciencia de la importancia de establecer marcos formativos homogéneos en educación superior, que permitirían la transferencia de conocimientos a los diversos ámbitos de la sociedad. La definición clara de las competencias que se deben adquirir en un proceso formativo doctoral es de vital importancia, dada las demandas del siglo XXI para con las universidades, donde el acento ya no está puesto en la formación de pregrado sino en la investigación de primer nivel, que sería el elemento identitario de estas instituciones (Nebot, 2009). El segundo, se refiere a las opiniones y expectativas de los doctorandos chilenos en educación sobre las competencias que han de adquirir al finalizar el proceso de formación que viven, las que están alineadas con las propuestas internacionales sobre lo que representa el grado académico de Doctor y los objetivos que tiene este en un contexto globalizado (Muñoz \& Araya, 2017; Villarroel \& Bruna, 2014). El tercer elemento, está referido a la escasa información emanada desde el Ministerio de Educación chileno sobre las competencias que se deben adquirir en un proceso de formación doctoral. La elección de las competencias está en manos de las instituciones que imparten estos programas, por lo que no es de extrañar la heterogeneidad evidenciada en los programas de doctorado en educación impartidos en Chile hasta el año 2018. Esta tríada es el artífice de la elaboración de un perfil de egreso estándar, que permita el análisis de los perfiles de egreso existentes, los cuales en este estudio se refieren a los programas dictados en el área de educación.

En definitiva, la falta de elementos relevantes en los perfiles de egreso y por tanto en la composición de un programa doctoral, pueden haber influido en los dictámenes de la comisión evaluadora, por lo que existiría una coherencia entre los años de 
acreditación recibidos y la calidad de los perfiles de egreso declarados. Esta situación explicaría porqué estos programas no alcanzaron la certificación de calidad en los tramos máximos establecidos por la Comisión Nacional de Acreditación.

En Chile, se iniciaron las discusiones sobre los postgrados avanzada la década del 2000, estando muy atrás ellas en comparación con Europa, lo que ha retrasado la regulación del nivel doctoral en el país (Corvalán, et. al, 2011). Para el año 2018 el Estado chileno, a través, de la Ley 21.091 intentó asegurar la existencia de programas doctorales de calidad (Ministerio de Educación, 2018a). Por este motivo el análisis presentado permite establecer las metas a alcanzar en el corto plazo, para así avanzar hacia una formación de nivel internacional para con los futuros doctores en educación chilenos. La definición clara de las competencias que ha de adquirir un futuro doctor es un reto para las instituciones dedicadas a dictar programas doctorales, pues esto influirá en las decisiones que se tomen tanto en los procesos formativos como en las líneas de investigación que se plantean en cada uno de estos.

En síntesis, los resultados obtenidos en este estudio esperan otorgar herramientas a las instituciones de educación superior sobre las decisiones que se deben tomar para alcanzar altos niveles de calidad en sus programas. El perfil de egreso, se constituye así, en una definición de cara a la sociedad sobre los procesos formativos que ofrece, donde se comprometen las competencias a desarrollar. Esto se traduciría eventualmente en el logro de los más altos estándares establecidos por los procesos de acreditación, considerando los niveles propuestos por la Comisión Nacional de Acreditación (2016b) para el caso chileno. La declaración de competencias tanto disciplinares como metodológicas, instrumentales y genéricas permitirán a los aspirantes al grado de Doctor conocer con claridad cuál será la formación con la que se compromete la institución por la que se han inclinado para su formación y permitirá la evaluación constante del perfil de egreso, en función de las demandas que emergen cada vez con más rapidez, haciéndose cargo de ellas de una manera más eficaz.

En conclusión, poseer una herramienta de gestión que permita elaborar, evaluar y eventualmente mejorar los perfiles de egreso del nivel doc- toral acercará a estos programas a la formación de profesionales más calificados y con las competencias que se requieren para el desarrollo de una determinada área de estudio. Finalmente, las limitaciones de este estudio están referidas al análisis de los doctorados pertenecientes al área de educación. Es por ello, que se espera replicar este estudio en otras áreas y colocar a disposición de la sociedad este instrumento analítico. Esto servirá para mejorar los procesos de definición de competencias en el nivel doctoral no solo en Chile, sino en países vecinos, los cuales comparten algunos elementos en lo que se refiere a la educación de cuarto nivel.

\section{Referencias}

Baeza, P. (2017). Diversidad y diferenciación en la oferta de programas de doctorado en Chile. Calidad en la Educación, 47, 179-214. https://doi.org/10.4067/ S0718-45652017000200179

Bernasconi, A. \& Rojas, F. (2004). Informe sobre la educación superior en Chile, 1980-2003. Santiago de Chile: Editorial Universitaria.

Cancino, V. \& Schmal, R. (2014). Sistema de Acreditación Universitaria en Chile: ¿Cuánto hemos avanzado? Estudios pedagógicos, 40(1), 41-60. https://doi. org/10.4067/S0718-07052014000100003

Chiappa, R., \& Muñoz García, A. (2015). Equidad y capital humano avanzado: Análisis sobre las políticas de formación de doctorado en Chile. Psicoperspectivas, 14(3), 17-30. https://doi.org/10.5027/psicoperspectivas-Vol14-Issue3-fulltext-621

Comisión Nacional de Acreditación \& Ministerio de Educación (2007). CNAP 1997-2007. El modelo chileno de acreditación de la educación superior. Santiago de Chile: Editora e Imprenta Maval.

Comisión Nacional de Acreditación (2016a). Aprueba texto refundido del Reglamento que fija el procedimiento para el desarrollo de los procesos de acreditación de programas de postgrado y especialidades del área de la salud. Santiago de Chile: Comisión Nacional de Acreditación.

Comisión Nacional de Acreditación (2016b). Propuesta Comité Consultivo de Postgrado: Operacionalización 
de Criterios de Evaluación para la Acreditación de Programas de Postgrado: Doctorado, Magíster Académico y Magíster Profesional. Santiago de Chile: Comisión Nacional de Acreditación.

Comisión Nacional de Acreditación (2018). Aprueba modificaciones al Reglamento que fija el procedimiento para el desarrollo de los procesos de acreditación de programas de postgrado y especialidades en el área de la salud. Santiago de Chile: Comisión Nacional de Acreditación. Recuperado de https://www.cnachile.cl/SiteAssets/Paginas/Acreditacion-Postgrado/002-4.pdf

Corvalán, J., Falabella, A., \& Rojas, M. T. (2011). El doctorado en educación: un ejemplo de desregulación en el campo de la educación superior en Chile. Calidad en la Educación, 34, 15-42. https://doi.org/10.4067/ S0718-45652011000100002

Espinoza, O., \& González, L. (2016). La regulación en la educación superior: una mirada sistémica considerando ámbitos y niveles de ejecución. En Lavados, H. y Berríos, R. (Eds.). Políticas para el desarrollo universitario: principios y evidencias, pp. 359-398. Santiago de Chile: Ediciones Universidad San Sebastián.

Fernández Fastuca, L., \& Wainerman, C. (2015). La dirección de tesis de doctorado: ¿una práctica pedagógica? Perfiles educativos, 37(148), 156-171. https://doi.org/10.1016/j.pe.2015.11.013

González, H., \& Jiménez, A. (2014). Inserción laboral de nuevos investigadores con grado de doctor en Chile. Journal of Technology Management \& Innovation, 9(4), 132-148. https://doi.org/10.4067/S071827242014000400010

González, J. A., Sarzoza, S. J., \& López, D. A. (2018). Aproximación metodológica a la metaevaluación de programas doctorales. Revista Venezolana de Gerencia, 23(1), 278-294.

Hawes, G. (2010). Glosario básico para la modernización curricular. Santiago de Chile: Universidad de Chile.

Horgué, C. (2012). La ordenación del Doctorado. Revista de Administración Pública, 189, 365-401. Recuperado de https://dialnet.unirioja.es/servlet/articulo?codigo $=4101084$

Jiménez-Ramírez, M. (2017). Los nuevos estudios de doctorado en España: avances y retos para su convergencia con Europa. Revista Iberoamericana de Educación Superior, 8 (21), 123-137. https://doi.org/10.22201/iisue.20072872e.2017.21.217

Knust, R., \& Gómez, S. (2009). La evaluación con enfoque por competencias: ¿Se implementa realmente la evaluación por competencias? Experiencias en Holanda y diferentes países de América Latina. Revista Electrónica de Desarrollo de Competencias, 3(1), 104-125.

Krauskopf, M. (2003). Indicadores cuantitativos de los doctorados conferidos en el país. ¿Falta de atención o expresión de subdesarrollo? Calidad en la edu cación, 18, 47-59. https://doi.org/10.31619/caledu. n18.385

Ley $N^{\circ}$ 21.091. Diario Oficial de la República de Chile, Santiago, Chile, 29 mayo de 2018.

Ley de Aseguramiento de la Calidad de la Educación N²0.129. Diario Oficial de la República de Chile, Santiago, Chile, 27 noviembre de 2006.

Ministerio de Educación (2018a). Base de datos Matrícula Histórica- Informe SIIES. Santiago de Chile: Ministerio de Educación.

Ministerio de Educación (2018b). Ley 21.091. Recuperado del sitio de Internet de la Biblioteca del Congreso Nacional de Chile: https://www.leychile.cl/Navegar?idNorma=1118991

Möller, I., \& Gómez, H. (2014). Coherencia entre perfiles de egreso e instrumentos de evaluación en carreras de educación básica en Chile. Calidad en la Educación, 41, 17-49. https://doi.org/10.4067/S071845652014000200002

Muñoz, D. R., \& Araya, D. H. (2017). Los desafíos de la evaluación por competencias en el ámbito educativo. Educação e Pesquisa, 43(4), 1073-1086. https://doi. org/10.1590/s1678-4634201706164230

Nebot, I. J. (2009). Introducción. El desafío de los programas de doctorado. En Agencia Nacional de Evaluación de la Calidad y Acreditación (Ed.). El doctorado: logros y desafíos (pp. 11-20). Madrid: ANECA LIBRO

Núñez Valdés, K. (2017). Evaluación de los aprendizajes sobre ciudadanía: meta evaluación de los instrumentos utilizados en el segundo ciclo básico chileno. Estudios pedagógicos, 43(2), 253-276. https://doi. org/10.4067/S0718-07052017000200014

Núñez-Valdés, K., \& González, J. (2019). Perfil de egreso doctoral: una propuesta desde el análisis documental y las expectativas de los doctorandos. IE Revista de investigación educativa de la REDIECH, 10(18), 161-175. https://doi.org/10.33010/ie_rie_rediech. v10i18.604

Silverman, E., Courgeau, D., Franck, R., Bijak, J., Hilton, J., Noble, J., \& Bryden, J. (2018). Methodological Inves tigations in Agent-Based Modelling: With Applications for the Social Sciences. New York: Springer 
Open. https://doi.org/10.1007/978-3-319-72408-9

Spronken-Smith, R., Brown, K., \& Mirosa, R. (2018). Employability and graduate attributes of doctoral graduates. In E. Bitzer, L. Frick, M. Fourie-Malherbe, K. Pyhältö (Eds.) Spaces, Journeys and new horizons for postgraduate supervision (pp. 233256). Stellenbosch: African Sun Media. https://doi. org/10.18820/9781928357810

Vera-Villarroel, P. (2010). Historia y estado actual de la formación doctoral en Chile. Revista Digital Universitaria, 11(5). Recuperado de http://www.revista. unam.mx/vol.11/num5/art47/index.html

Villarroel, V., \& Bruna, D. (2014). Reflexiones en torno a las competencias genéricas en educación superior: Un desafío pendiente. Psicoperspectivas, 13(1), 23-34. https://doi.org/10.5027/psicoperspectivas-Vol13-Issue1-fulltext-335

Weijden, I., Teelken, C., de Boer, M., \& Drost, M. (2016). Career satisfaction of postdoctoral researchers in relation to their expectations for the future. The International Journal of Higher Education Research, 72(1), 25 40. https://doi.org/10.1007/s10734-015-9936-0

RIDU / Revista Digital de Investigación en Docencia Universitaria / e-ISSN: 2223-2516

(C) Los autores. Este artículo es publicado por la Revista Digital de Investigación en Docencia Universitaria del Área de Institutional Research and Effectiveness de la Dirección de Aseguramiento de la Calidad, Universidad Peruana de Ciencias Aplicadas. Este es un artículo de acceso abierto, distribuido bajo los términos de la LicenciaCreativeCommons Atribución-CompartirIgual 4.0 Internacional.( http://creativecommons.org/licenses/by-sa/4.0/), que permite el uso no comercial, distribución y reproducción en cualquier medio, siempre que la obra original sea debidamente citada. 